\title{
RELIABLE PHASE STABILITY ANALYSIS FOR CUBIC EQUATION OF STATE MODELS
}

\author{
JAMES Z. HUA ${ }^{\mathrm{a}}$, JOAN F. BRENNECKE ${ }^{\mathrm{b}}$ and MARK A. STADTHERR ${ }^{\mathrm{b} 1}$
}

${ }^{a}$ Department of Chemical Engineering, University of Illinois, 600 S. Mathews Ave., Urbana, IL 61801 USA

bDepartment of Chemical Engineering, University of Notre Dame, Notre Dame, IN 46556 USA

\begin{abstract}
The reliable prediction of phase stability is a challenging computational problem in chemical process simulation, optimization and design. The phase stability problem can be formulated either as a minimization problem or as an equivalent nonlinear equation solving problem. Conventional solution methods are initialization dependent, and may fail by converging to trivial or nonphysical solutions or to a point that is a local but not global minimum. Thus there has been considerable recent interest in developing more reliable techniques for stability analysis. In this paper we demonstrate, using cubic equation of state models, a technique that can solve the phase stability problem with complete reliability. The technique, which is based on interval analysis, is initialization independent, and if properly implemented provides a mathematical guarantee that the correct solution to the phase stability problem has been found.
\end{abstract}

\section{INTRODUCTION}

The determination of phase stability, i.e., whether or not a given phase will split into multiple phases, is a key step in phase equilibrium calculations, and thus in the simulation and design of a wide variety of processes, especially those involving separation operations such as distillation and extraction. The phase stability problem is frequently formulated in terms of the tangent plane condition (Baker et al., 1982). Minima in the tangent plane distance are sought, usually by solving a system of nonlinear equations for the stationary points (Michelsen, 1982). If any of these yield a negative tangent plane distance, indicating that the tangent plane intersects (or lies above) the Gibbs energy of mixing surface, the phase is unstable. The difficulty lies in that, in general, given any arbitrary equation of state or activity coefficient model, most computational methods cannot find with complete certainty all the stationary points, and thus there is no guarantee that the phase stability problem has been correctly solved.

Standard methods (e.g., Michelsen, 1982) for solving the phase stability problem typically rely on the use of multiple initial guesses, carefully chosen in an attempt to locate all stationary points in the tangent plane distance function. However, these methods offer no guarantee that the global minimum in the tangent plane distance has been found. Because of the difficulties that thus arise, there has been significant recent interest in the development of more reliable methods for solving the phase stability problem (e.g., Sun and Seider, 1995; Eubank et al. ,1992; Wasylkiewicz et al., 1993; McDonald and Floudas, 1995). For example, Sun and Seider (1995) apply a homotopycontinuation method, which will often find all the stationary points, and is easier to initialize than Michelsen's approach. However, their technique is still initialization dependent and provides no theoretical guarantees that all stationary points have been found. The "area" method of Eubank et al. (1992), which is based on exhaustive search over a grid, can also be very reliable. They suggest that a course grid be used first to find the approximate location of solutions. Then, regions appearing not to contain a solution are arbitrarily eliminated from consideration and the search continues with a finer grid in the remaining regions. However, there is no mathematical guarantee provided that the regions eliminated do not contain solutions. McDonald and Floudas (1995) show that for certain activity coefficient models, the phase stability problem can be reformulated to make it amenable to solution by powerful global optimization techniques, which do guarantee that the correct answer is found. However, in general there appears to remain a need for an efficient general-purpose method that can perform phase stability calculations with mathematical certainty for any arbitrary equation of state (EOS) or activity coefficient model.

An alternative approach that satisfies these needs has been suggested by Stadtherr et al. (1995) and Hua et al. (1996), and applied to both activity coefficient models and a simple cubic EOS (Van der Waals equation). This

\footnotetext{
${ }^{1}$ Author to whom all correspondence should be addressed.
} 
technique, which is based on interval analysis, in particular the use of an interval Newton/generalized bisection algorithm, is initialization independent and can solve the phase stability problem with mathematical certainty. In this paper we seek to extend the application of this technique to cubic EOS models in general. Though the technique developed is general-purpose, the applications presented focus on the Peng-Robinson (PR) and Soave-Redlich-Kwong (SRK) models.

\section{PHASE STABILITY ANALYSIS}

The determination of phase stability is often done using tangent plane analysis (Baker et al., 1982; Michelsen, 1982). A phase at specified $T, P$, and feed mole fraction $\mathbf{z}$ is unstable if the Gibbs energy of mixing versus composition surface $m=\Delta g^{\mathrm{M}} / R T$ ever falls below a plane tangent to the surface at $\mathbf{z}$. That is, if the tangent plane distance

$$
D(x)=m(x)-m_{0}-\sum_{i=1}^{n}\left(\frac{\partial m}{\partial x_{i}}\right)_{0}\left(x_{i}-z_{i}\right)
$$

is negative for any composition $\mathbf{x}$, the phase is unstable. The subscript zero indicates evaluation at $\mathbf{x}=\mathbf{z}$, and $n$ is the number of components. A common approach for determining if $D$ is ever negative is to minimize $D$ subject to the mole fractions summing to one. It is readily shown that the stationary points in this optimization problem can be found by solving the system of nonlinear equations:

$$
\begin{gathered}
{\left[\left(\frac{\partial m}{\partial x_{i}}\right)-\left(\frac{\partial m}{\partial x_{i}}\right)_{0}\right]-\left[\left(\frac{\partial m}{\partial x_{n}}\right)-\left(\frac{\partial m}{\partial x_{n}}\right)_{0}\right]=0, \quad i=1, \ldots, n-1} \\
1-\sum_{i=1}^{n} x_{i}=0
\end{gathered}
$$

If an EOS model for $m$ is used, so that $m$ is expressed in terms of $\mathbf{x}$ and $v$, the molar volume of the mixture, then (2) and (3) must be solved simultaneously with the EOS. The model used here is the generalized cubic EOS

$$
P-\frac{R T}{v-b}+\frac{a}{v^{2}+u b v+w b^{2}}=0
$$

given by Reid et al. (1987). With the appropriate choice of $u$ and $w$, common models such as PR $(u=2, w=-1)$ and SRK $(u=1, w=0)$ may be obtained. For all the example problems considered here, standard mixing rules, namely $b=\Sigma x_{i} b_{i}$ and $a=\Sigma \Sigma x_{i} x_{j} a_{i j}$, are used.

The $(n+1) \times(n+1)$ system given by equations (2)-(4) above has a trivial root at $\mathbf{x}=\mathbf{z}$ and $v=v_{0}$ and frequently has multiple nontrivial roots as well. Thus conventional equation solving techniques such as Newton's method may fail by converging to the trivial root or give an incorrect answer to the phase stability problem by converging to a stationary point that is not the global minimum of $D$. This is aptly demonstrated by the experiments of Green et al. (1993), who show that the pattern of convergence from different initial guesses demonstrates a complex fractal-like behavior for even very simple models. We demonstrate here the use of an interval Newton/generalized bisection method for solving the system (2)-(4). The method requires no initial guess, and will find with certainty all the stationary points of $D$.

\section{INTERVAL COMPUTATIONS}

A real interval, $X$, is defined as a set of real numbers lying between (and including) upper and lower bounds; i.e., $X=[a, b]=\{x \in \mathfrak{R} \mid a \leq x \leq b\}$, where $a, b \in \mathfrak{R}$ and $a \leq b$. A real interval vector $\mathbf{X}=\left(X_{1}, X_{2}, \ldots, X_{n}\right)^{\mathrm{T}}$ has $n$ real interval components and since it can be interpreted geometrically as an $n$-dimensional rectangle, is frequently referred to as a box. Note that in this section lower case quantities are real numbers and upper case quantities are intervals. Several good introductions to computation with intervals are available, including recent monographs by Neumaier (1990) and Hansen (1992). 
Of particular interest here are interval Newton/generalized bisection (IN/GB) methods. These techniques provide the power to find with confidence all solutions of a system of nonlinear equations (Neumaier, 1990; Kearfott and Novoa, 1990), and to find with total reliability the global minimum of a nonlinear objective function (Hansen, 1992), provided only that upper and lower bounds are available for all variables. Efficient techniques for implementing IN/GB are a relatively recent development, and thus such methods have not yet been widely applied. Schnepper and Stadtherr (1990) have suggested the use of this method for solving chemical process modeling problems, and recently described an implementation (Schnepper and Stadtherr, 1996). Balaji et al. (1995) have also successfully applied the method to chemical engineering problems.

Consider the solution of the system of real nonlinear equations $\mathbf{f}(\mathbf{x})=\mathbf{0}$, where it is desired to find all solutions in an specified initial interval $\mathbf{X}^{(0)}$. The basic iteration step in interval Newton methods is, given an interval $\mathbf{X}^{(k)}$, to solve the linear interval equation system

$$
\mathrm{F}^{\prime}\left(\mathbf{X}^{(k)}\right)\left(\mathbf{N}^{(k)}-\mathbf{x}^{(k)}\right)=-\mathbf{f}\left(\mathbf{x}^{(k)}\right)
$$

for a new interval $\mathbf{N}^{(k)}$, where $k$ is an iteration counter, $\mathrm{F}^{\prime}\left(\mathbf{X}^{(k)}\right)$ is an interval extension of the real Jacobian $\mathrm{f}^{\prime}(\mathbf{x})$ of $\mathbf{f}(\mathbf{x})$ over the current interval $\mathbf{X}^{(k)}$, and $\mathbf{x}^{(k)}$ is a point in the interior of $\mathbf{X}^{(k)}$, usually taken to be the midpoint. The interval extension $\mathrm{F}^{\prime}\left(\mathbf{X}^{(k)}\right)$ of the Jacobian is determined by substituting the interval $\mathbf{X}^{(k)}$ for $\mathbf{x}$ in the expression $\mathrm{f}^{\prime}(\mathbf{x})$ for the real Jacobian, and performing interval operations in place of real operations. It can be shown (Moore, 1966) that any root $\mathbf{x}^{*}$ of the set of equations that is within the current interval, i.e. $\mathbf{x}^{*} \in \mathbf{X}^{(k)}$, is also contained in the newly computed interval $\mathbf{N}^{(k)}$. This suggests that the next iteration for $\mathbf{X}$ should be the intersection of $\mathbf{X}^{(k)}$ with $\mathbf{N}^{(k)}$, i.e. $\mathbf{X}^{(k+1)}=\mathbf{X}^{(k)} \cap \mathbf{N}^{(k)}$. There are various interval Newton methods, which differ in how they determine $\mathbf{N}^{(k)}$ from equation (5) and thus in the tightness with which $\mathbf{N}^{(k)}$ encloses the solution set of (5).

While the iteration scheme discussed above can be used to tightly enclose a solution, what is of most significance here is the power of (5) to provide a test of whether a solution exists within a given interval and whether it is a unique solution. For several techniques for finding $\mathbf{N}^{(k)}$ from (5), it can be proven (e.g., Neumaier, 1990) that if $\mathbf{N}^{(k)}$ is totally contained within $\mathbf{X}^{(k)}$, i.e. $\mathbf{N}^{(k)} \subset \mathbf{X}^{(k)}$, then there is a unique zero of the set of nonlinear equations $\mathbf{f}(\mathbf{x})=\mathbf{0}$ in $\mathbf{X}^{(k)}$, and furthermore that Newton's method with real arithmetic will converge to that solution starting from any point in $\mathbf{X}^{(k)}$. Thus, if $\mathbf{N}^{(k)}$ is determined using one of these techniques, the computation can be used as a root inclusion test for any interval $\mathbf{X}^{(k)}$ : 1) If $\mathbf{X}^{(k)}$ and $\mathbf{N}^{(k)}$ do not intersect, i.e., $\mathbf{X}^{(k)} \cap \mathbf{N}^{(k)}=\varnothing$, then there is no root in $\mathbf{X}^{(k)}$. 2) If $\mathbf{N}^{(k)}$ is totally contained in $\mathbf{X}^{(k)}$, then there is exactly one root in $\mathbf{X}^{(k)}$ and Newton's method with real arithmetic will find it. Otherwise, 3) no conclusion can be drawn. In the last case, one could then repeat the root inclusion test on the next interval Newton iterate $\mathbf{X}^{(k+1)}$, assuming it is sufficiently smaller than $\mathbf{X}^{(k)}$, or one could bisect $\mathbf{X}^{(k+1)}$ and repeat the root inclusion test on the resulting intervals. This is the basic idea of IN/GB methods. If $\mathbf{f}(\mathbf{x})=\mathbf{0}$ has a finite number of real solutions in the specified initial box, a properly implemented IN/GB method can find with mathematical certainty any and all solutions to a specified tolerance, or can determine with mathematical certainty that there are no solutions in the given box (Kearfott and Novoa, 1990; Kearfott, 1990). The technique used here for computing $\mathbf{N}^{(k)}$ from (5) is the preconditioned Gauss-Seidel-like technique developed by Hansen and Sengupta (1981). A detailed step-by-step description of the IN/GB algorithm used here is given by Schnepper and Stadtherr (1996).

The set of equations (2)-(4) that must be solved involves $n+1$ variables, the $n$ component mole fractions and the molar volume. For the mole fractions, the initial box $\mathbf{X}^{(0)}=[\mathbf{0 , 1}]$ is suitable. In practice the initial lower bound is set to an arbitrarily small positive number $\varepsilon\left(10^{-10}\right.$ was used) to avoid taking the logarithm of zero in subsequent calculations. This can be done without the loss of reliability providing a sufficiently small value of $\varepsilon$ is used. The lower limit on the molar volume was taken to be the smallest pure component size parameter $b$, and the upper bound was taken to be the ideal gas molar volume for the $T$ and $P$ under investigation. Although it is possible to have compressibility factors greater than one at very high $P_{\mathrm{r}}$, this was deemed satisfactory for the range of $T_{\mathrm{r}}$ and $P_{\mathrm{r}}$ explored below. Our implementation of the IN/GB method for the phase stability problem is based on appropriately modified routines from the packages INTBIS (Kearfott and Novoa, 1990) and INTLIB (Kearfott et al., 1994).

\section{RESULTS}

To test this initial implementation of IN/GB to solve phase stability problems for cubic equations of state, several different mixtures have been used. Results for three mixtures using either the SRK or PR model are presented here.

Problem 1. This is a mixture of hydrogen sulfide (1) and methane (2) at $190 \mathrm{~K}$ and 40.53 bar. The SRK 
model was used with parameters calculated from $T_{\mathrm{c} 1}=$ $373.2 \mathrm{~K}, P_{\mathrm{c} 1}=89.4$ bar, $\omega_{1}=0.1, T_{\mathrm{c} 2}=190.6 \mathrm{~K}, P_{\mathrm{c} 2}=$ 46.0 bar, $\omega_{2}=0.008$, and a binary interaction parameter $k_{12}=0.08$. Feeds of $z_{1}=0.0185$ and $z_{1}=0.5$ were considered. Results are presented in Table 1, which shows the roots (stationary points) found, and the value of the tangent plane distance $D$ at each root. Both feeds are unstable, as indicated by the negative values found for $D$. For the $z_{1}=0.5$ case, our results are consistent with those given by Sun and Seider (1995) for this problem. For the $z_{1}=0.0185$ case, a plot of $D$ vs. $x_{1}$ is shown in Figs. 1 and 2. Note that, as pointed out by Michelsen (1982), if one uses a locally convergent solver, with pure $\mathrm{CH}_{4}\left(x_{1}=0\right)$ as the initial guess, convergence will be to the trivial solution at $x_{1}$ $=z_{1}=0.0185$. And if pure $\mathrm{H}_{2} \mathrm{~S}\left(x_{1}=1\right)$ is the initial guess, convergence will be to the local, but not global,

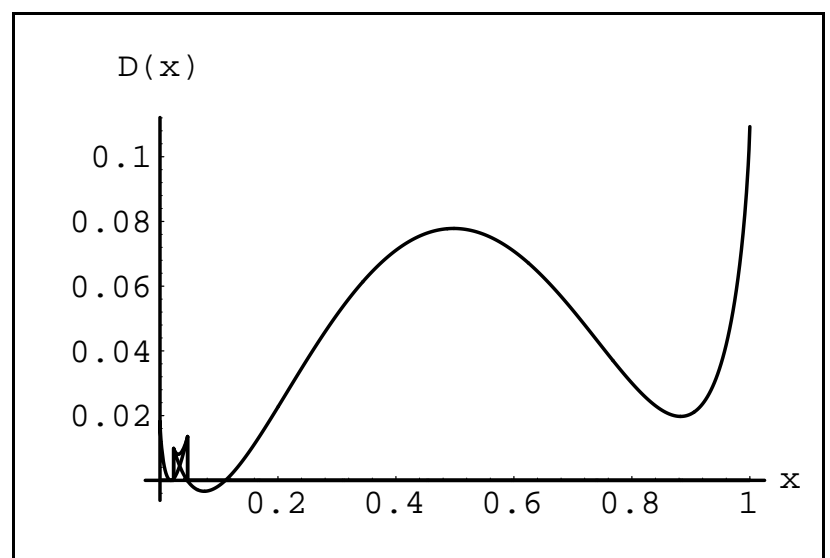

Figure 1. $D$ vs. $x_{1}$ for Problem 1 with $z_{1}=0.0185$. Vertical lines demarcate region of multiple real volume roots (see Fig. 2). minimum at $x_{1}=0.8826$. Using only these initial guesses would lead to the incorrect conclusion that the mixture is stable. This is indicative of the importance of the initialization strategy when conventional methods are used, An important advantage of the IN/GB approach described here is that it eliminates the initialization problem, since it is initialization independent. In this case, it finds all the stationary points, including the global minimum at $x_{1}=0.0751$. Note also that the presence of multiple real volume roots does not present any difficulty, since the solver simply finds all roots for the given system.

Also included in Table 1 are the number of root inclusion tests performed in the computation, the level reached in the binary tree generated in the bisection, and the total CPU time on an HP 9000/735 workstation. We would expect standard approaches to the phase stability problem to be faster, but these methods do not reliably solve the problem in all cases. Thus, as one might expect, to obtain guaranteed reliability some premium must be paid in terms of computation time. It should be noted that earlier experience (Stadtherr et al., 1995) in applying the IN/GB approach to liquid/liquid phase stability problems using the NRTL equation, indicated that the computational efficiency of IN/GB compared favorably with the model-specific technique of McDonald and Floudas (1995), which also offers guaranteed reliability on the NRTL problem. It should also be noted that

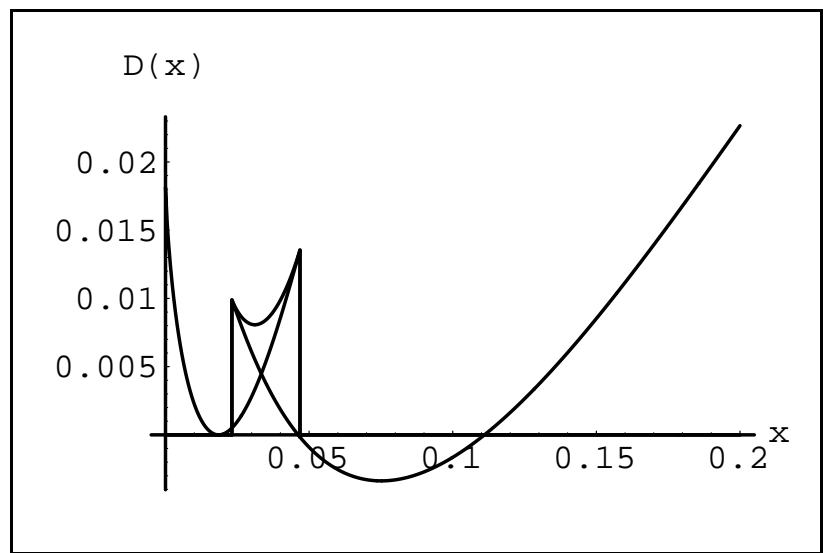

Figure 2. Enlargement of Fig. 1 showing region near the origin. Vertical lines demarcate region of multiple real volume roots. this is an initial implementation of IN/GB for the phase stability problem with the generalized cubic equation of state, and we anticipate that significant improvements can be made in its computational efficiency.

Problem 2. This is a mixture of methane (1) and propane (2) at $277.6 \mathrm{~K}$ and $50 \mathrm{bar}$. The SRK model was used with parameters calculated from methane data given above, $T_{\mathrm{c} 2}=369.8 \mathrm{~K}, P_{\mathrm{c} 2}=42.5 \mathrm{bar}, \omega_{2}=0.152$, and a binary interaction parameter $k_{12}=0.029$. Feeds of $z_{1}=0.1,0.4$, and 0.6 were considered, with results shown in Table 1. The first feed is stable and the others unstable, which is consistent with known phase behavior (Prausnitz, et. al., 1986). Several other values of $T, P$, and $z_{1}$ have also been tried and correct results obtained.

Problem 3. This is a mixture of nitrogen (1) and ethane (2) at $270 \mathrm{~K}$ and 76 bar. The PR model was used with parameters calculated from $T_{\mathrm{c} 1}=126.2 \mathrm{~K}, P_{\mathrm{c} 1}=33.9 \mathrm{bar}, \omega_{1}=0.04, T_{\mathrm{c} 2}=305.4 \mathrm{~K}, P_{\mathrm{c} 2}=48.8 \mathrm{bar}, \omega_{2}=0.098$, and a binary interaction parameter $k_{12}=0.08$. Feeds of $z_{1}=0.1,0.3$, and 0.65 were considered, with results shown in Table 1. The second feed is unstable and the others stable, which is consistent with known phase behavior (Prausnitz, et. al., 1986). Several other values $z_{1}$ have also been tried and correct results obtained.

While the results presented here are for binary mixtures only, the method is applicable to problems with a larger number of components, and tests involving ternary and larger systems are currently under way. 
Table 1. Roots (stationary points) found and performance

\begin{tabular}{|c|c|c|c|c|c|}
\hline Feed $\left(z_{1}, z_{2}\right)$ & $\operatorname{Roots}\left(x_{1}, x_{2}, v\left[\mathrm{~cm}^{3} / \mathrm{mol}\right]\right)$ & $D$ & $\begin{array}{l}\text { Root } \\
\text { inclusion } \\
\text { tests }\end{array}$ & $\begin{array}{l}\text { Level } \\
\text { reached in } \\
\text { binary tree }\end{array}$ & $\begin{array}{l}\text { CPU Time } \\
\text { HP 9000/ } \\
735 \text { (sec) }\end{array}$ \\
\hline
\end{tabular}

Problem 1:

$0.5,0.5$

$\begin{array}{ll}0.5,0.5,41.32 & 0.0 \\ 0.8819,0.1181,36.60 & -0.0569 \\ 0.0746,0.9254,64.44 & -0.0825 \\ 0.0184,0.9816,207.5 & -0.0793 \\ 0.0311,0.9689,114.9 & -0.0721\end{array}$

$0.0185,0.9815$

$\begin{array}{ll}0.0185,0.9815,207.48 & 0.0 \\ 0.8826,0.1174,36.59 & 0.0197 \\ 0.0751,0.9249,64.36 & -0.0034 \\ 0.0311,0.9689,115.0 & 0.0081 \\ 0.4978,0.5022,41.36 & 0.0778\end{array}$

Problem 2:

$\begin{array}{llllll}0.1,0.9 & 0.1,0.9,86.71 & 0.0 & 2029 & 19 & 1.01 \\ 0.4,0.6 & 0.4,0.6,89.46 & 0.0 & 4459 & 24 & 2.24 \\ & 0.8654,0.1346,378.4 & -0.153 & & & \\ & 0.5516,0.4484,115.3 & 0.0106 & & & \\ & & & & \\ 0.6,0.4 & 0.7058,0.2942,313.0 & -0.0065 & 3948 & 20 & \\ & 0.1928,0.8072,86.07 & -0.223 & & & \\ & 0.6,0.4,216.5 & 0.0 & & \end{array}$

Problem 3:

$\begin{array}{llllll}0.1,0.9 & 0.1,0.9,71.11 & 0.0 & 1932 & 18 & 0.88 \\ 0.3,0.7 & 0.3,0.7,112.3 & 0.0 & 4813 & 23 & 2.24 \\ & 0.4893,0.5107,198.3 & -0.0138 & & & \\ 0.1767,0.8233,78.18 & -0.0069 & & & \\ 0.65,0.35 & 0.65,0.35,238.4 & 0.0 & 2828 & 20 & 1.21\end{array}$

\section{CONCLUSIONS AND SIGNIFICANCE}

Results demonstrate that the interval Newton/generalized bisection algorithm can solve phase stability problems for a generalized cubic equation of state model efficiently and with complete reliability. This work represents an entirely new method for solving these problems, a method that can guarantee with mathematical certainty that the correct solutions are found, thus eliminating computational problems that are frequently encountered with currently available techniques. The method is initialization independent; it is also model independent and can be applied in connection with other equations of state or with activity coefficient models.

\section{ACKNOWLEDGMENTS}

This work was supported in part by the Environmental Protection Agency and by the National Science Foundation under Grant CTS-9522835. 


\section{REFERENCES}

Baker, L. E., A. C. Pierce, and K. D. Luks, Gibbs energy analysis of phase equilibria. Soc. Petrol. Engrs. J., 22, 731-742 (1982).

Balaji, G. V., J. D. Seader, J. J. Chen, and S. Sharma, Application of interval-Newton method to chemical engineering problems. AIChE Symp. Ser., 91, No. 304, 364-367 (1995).

Eubank, P. T., A. E. Elhassan, M. A. Barrufet, and W. B. Whiting, Area method for prediction of fluid phase equilibria. Ind. Eng. Chem. Res., 31, 942-949 (1992).

Green, K. A., S. Zhou, and K. D. Luks, The fractal response of robust solution techniques to the stationary point problem. Fluid Phase Equilib., 84, 49-78 (1993).

Hansen, E. R., Global Optimization Using Interval Analysis. M. Dekkar, New York (1992).

Hansen, E. R. and S. Sengupta, Bounding solutions of systems of equations using interval analysis. BIT, 21, $203-211$ (1981).

Hua, J. Z., J. F. Brennecke, and M. A. Stadtherr, Reliable prediction of phase stability using an interval-Newton method. Fluid Phase Equilib., in press (1996).

Kearfott, R. B., Interval arithmetic techniques in the computational solution of nonlinear systems of equations: Introduction, examples, and comparisons. Lectures in Applied Mathematics, 26, 337-357 (1990).

Kearfott, R. B. and M. Novoa III, INTBIS, a portable interval Newton/bisection package. ACM Trans. Math. Softw., 16, 152-157 (1990).

Kearfott, R. B., M. Dawande, and K. Du, Algorithm 737: INTLIB: a portable Fortran 77 interval standard-function library. ACM Trans. Math. Softw, 20, 447-59 (1994).

McDonald, C. and C. A. Floudas, Global optimization for the phase stability problem. AIChE J., 41, 1798-1814 (1995).

Michelsen, M. L., The isothermal flash problem. Part I: Stability. Fluid Phase Equilib., 9, 1-19 (1982).

Moore, R. E., Interval Analysis. Prentice-Hall, Englewood Cliffs, NJ (1966).

Neumaier, A., Interval Methods for Systems of Equations. Cambridge University Press, Cambridge (1990).

Prausnitz, J. M., R. N. Lichtenthaler, and E. Gomes de Azevedo, Molecular Thermodynamics of Fluid-Phase Equilibria. Prentice-Hall, Englewood Cliffs, NJ (1986).

Reid, R. C., J. M. Prausnitz, and B. E. Poling, The Properties of Gases and Liquids (4th Ed.). McGraw-Hill, New York (1987).

Schnepper, C. A. and M. A. Stadtherr, On using parallel processing techniques in chemical process design, presented at AIChE Annual Meeting, Chicago, Nov. (1990).

Schnepper, C. A. and M. A. Stadtherr, Robust process simulation using interval methods. Comput. Chem. Eng., 20, 187-199 (1996).

Stadtherr, M. A., C. A. Schnepper, and J. F. Brennecke, Robust phase stability analysis using interval methods. AIChE Symp. Ser., 91, No. 304, 356-359 (1995).

Sun, A. C. and W. D. Seider, Homotopy-continuation for stability analysis in the global minimization of the Gibbs free energy. Fluid Phase Equilib, 103, 213-249 (1995).

Wasylkiewicz, S. K., L. N. Sridhar, M. F. Malone, and M. F. Doherty, Gibbs tangent plane analysis for complex liquid mixtures: A global algorithm. Presented at AIChE Annual Meeting, St. Louis, November (1993). 